\section{Rescue Treatment with Terlipressin for Persistent Pulmonary Hypertension and Refractory Shock in a Preterm Infant}

Persistent pulmonary hypertension of the newborn (PPHN) affects about 2/1000 newborn. The mainstay of therapy is supportive high frequency oscillatory ventilation (HFOV) and inhaled nitric oxide (NO), which have decreased the need for Extracorporeal membrane oxygenation (ECMO) in these patients [1].However, still there is significant mortality and affected infants are at risk of long-term neurological impairment. We present the case of a preterm infant with severe PPHN and shock that failed all available therapies and was successfully rescued with the administration of terlipressin.

A 33-week male preterm baby (birthweight $2010 \mathrm{~g}$ ) was admitted to our neonatal intensive care unit. Soon after birth he developed respiratory distress and hypoxemia. He was intubated and a surfactant dose was administered but without significant improvement. An echocardiogram showed a structurally normal heart and a near-systemic pulmonary artery systolic pressure (PASP) of 55-60 $\mathrm{mmHg}$. HFOV and inhaled NO $(20 \mathrm{ppm})$ were then initiated. The infant was hypotensive (Mean arterial pressure, MAP $<5$ th percentile) requiring inotropic support with dopamine and dobutamine. At 36 hours of life, the infant remained hypotensive and with oxygenation failure (oxygenation index 31). Sequential echocardiograms showed supra-systemic PASP (80$100 \mathrm{mmHg}$ ), right heart failure and pure right-to-left shunts. Sildenafil was added and continuous prostaglandin E1 infusion was started to keep the ductus open and support cardiac output. Adequate MAP could not be maintained despite escalating inotropic support: norepinephrine (up to $0.8 \mu \mathrm{g} / \mathrm{kg}$ / min), epinephrine (up to $0.8 \mu \mathrm{g} / \mathrm{kg} / \mathrm{min}$ ) and hydrocortisone $(1 \mathrm{mg} / \mathrm{kg} / 6 \mathrm{~h})$. In this context, bosentan $(2 \mathrm{mg} / \mathrm{kg} / 12 \mathrm{~h})$ was added but neither improvement in pulmonary hypertension nor oxygenation was observed. Continuous epoprostenol infusion was initiated at 40 hours of life because of persistent right heart failure and shock. Pulmonary hypertension partially responded to this therapy with improved oxygenation (OI change from 40 to 24) but it was impossible to maintain systemic MAP at maximal inotropic support (vasoactiveinotropic score: 131). A contact with two ECMO transport teams was made at 2 nd day of life but ECMO was considered not indicated because of prematurity and a poor expected survival. At 4th day of life, the child was in profound shock and it was decided, in agreement with parents, to use compassionate rescue treatment with terlipressin. A $5 \mu \mathrm{g} / \mathrm{kg}$ bolus dose was administered followed by $10 \mu \mathrm{g} / \mathrm{kg} / \mathrm{h}$ continuous infusion. Terlipressin rapidly raised MAP to 60 $\mathrm{mmHg}$, allowing escalation of epoprostenol up to $60 \mathrm{ng} / \mathrm{kg} /$ min. PASP decreased from 85 to $40 \mathrm{mmHg}$ with improvement in oxygenation (OI from 38 to 15). In the next 24 hours, he developed left to right ductal shunt and he was progressively weaned from pulmonary vasodilators. Terlipressin was maintained for 48 hours and then tapered gradually $(1 \mu \mathrm{g} / \mathrm{kg} / \mathrm{h})$ allowing the infant to be weaned from catecholamines ( 7 th day of life) and finally extubated at 12 th day of life. The child was discharged home without any clinically significant sequelae. At 2 years follow up, the child has age-appropriate developmental status.

With terlipressin we observed a rapid raise in MAP with a marked improvement in pulmonary hypertension and oxygenation, allowing tapering of catecholamines. Terlipressin has been used to treat catecholamine-resistant vasodilatory shock in adults, showing restoration of arterial blood pressure. Terlipressin has also been used in pediatric and neonatal refractory shock with unclear clinical benefits [2,3]. Unlike adults, pure vasodilatory shock is uncommon in the pediatric and neonatal population and in consequence excessive vasoconstriction can induce tissue ischemia and worsen heart function and is not generally recommended.

Terlipressin is a vasopressin analogue with long half-life that exerts effects via V1 and V2 receptors. The main clinical effect is mediated by V1-receptor that causes smooth muscle contraction and induces a potent increase in systemic vascular resistance (SVR) and blood pressure.Terlipressin is reported to increase SVR without a concomitant increase in pulmonary vascular resistance (PVR) [4]. In fact terlipressin may induce direct pulmonary vasodilatation via NO-release and lower pulmonary arterial pressure. Terlipressin-induced increase in SVR also improves coronary perfusion and right heart function contributing to increased pulmonary blood flow and oxygenation; which is newborn infants may be further facilitated by reversal of right to left intra-cardiac shunts as a consequence of increased MAP/PAP ratio [5].

Successful use of terlipressin in term infants with different causes of PPHN such as congenital diaphragmatic hernia has been reported [6]. However, its use has not been reported in preterm infants, in whom refractory PPHN is uncommon. In our patient, given the potentially reversible nature of idiopathic PPHN we decided to exhaust all therapeutic measures and try rescue treatment with terlipressin. We observed improved blood pressure, heart function, and oxygenation. During the infusion, cardio-respiratory monitoring, NIRS, ion control, echocardiography, troponin and serial clinical evaluations were used to monitor side effects. We only observed transient skin vasoconstriction and discoloration without development of skin necrosis or evidence of new-organ failure.

In conclusion we believe that terlipressin may be considered as a salvage therapy in severe PPHN and refractory hypotension when ECMO is not available or it is considered contraindicated.

Ignacio Oulego-Erroz, ${ }^{1,2}$ SANDra Terroba-Seara,
Leticia Castanon-LoPez ${ }^{3}$ AND
Antonio Rodriguez-Nunez
${ }^{4}$ Pediatric Cardiology and Pediatric Intensive Care Unit,

VOLUME 57-SEPTEMBER 15, 2020 
Complejo Asistencial Universitario de Leon; ${ }^{2}$ IBIOMED (Biomedicine Institute of Leon, University of Leon, Leon, ${ }^{3}$ Neonatal Intensive Care Unit,Complejo Asistencial Universitario de Leon; and

${ }^{4}$ Pediatric Intensive, Intermediate and Palliative Care Section, ComplejoHospitalarioUniversitario de Santiago, Santiago de Compostela; Spain.

*sandra_viveiro@hotmail.com

\section{REFERENCES}

1. Fuloria M, Aschner JL. Persistent pulmonary hypertension of the newborn.Semin Fetal Neonatal Med. 2017;22:220-6.

2. Masarwa R, Paret G, Perlman A, Reif S, Raccah BH, Matok I. Role of vasopressin and terlipressin in refractory shock compared to conventional therapy in the neonatal and pediatric population: A systematic review, metaanalysis, and trial sequential analysis. Crit Care. 2017;21:1.
3. Rodríguez-Núñez A, López-Herce J, Gil-Antón J, Hernández A, Rey C, RETSPED Working Group of the Spanish Society of Pediatric Intensive Care. Rescue treatment with terlipressin in children with refractory septic shock: A clinical study. Crit Care. 2006;10:R20.

4. Radicioni M, Troiani S, Camerini PG. Effects of terlipressin on pulmonary artery pressure in a septic cooled infant: an echocardiographic assessment. J Perinatol. 2012;32:89-5.

5. Evora PR, Pearson PJ, Schaff HV. Arginine vasopressin induces endothelium-dependent vasodilatation of the pulmonary artery. V1-receptor-mediated production of nitric oxide.Chest. 1993;103:1241-5.

6. Stathopoulos L, Nicaise C, Michel F, Thomachot L, Merrot T, Lagier P, et al. Terlipressin as rescue therapy for refractory pulmonary hypertension in a neonate with a congenital diaphragmatic hernia. J Pediatr Surg. 2011;46:e19-21.

\section{Bartter Syndrome Masquerading as Acute Kidney Injury in a Neonate}

Infants and children with Bartter syndrome present with polyuria and polydipsia, whereas older children present with constipation, salt craving and muscle cramps. The symptomatology is mainly due to renal concentrating defect [1]. This disorder is characterized by hypokalemia, hypochloremia, hypercalciuria, salt wasting with metabolic alkalosis.

A 10-day-old male child born out of third degree consanguineous marriage presented with severe respiratory distress. The antenatal history was uneventful. The neonate was suspected as late-onset sepsis and appropriate management was started. The investigations showed normal counts, C-reactive protein (CRP) levels and urine examination. The condition deteriorated further and required mechanical ventilation. The baby was started on intravenous piperacillin-tazobactam and amikacin, but antibiotics were stopped after 7 days as blood culture sensitivity was negative.

The baby's condition gradually improved and was weaned from the ventilator after 8 days. Renal parameters, urine output and leucocyte counts were monitored regularly and remained normal. The blood gases were all normal. The child was started on breast feeds on day 32 of life and was under observation for proper feed establishment and weight gain. On day 48, the infant developed decreased urine output along with respiratory distress for second time. Investigations showed normal leucocyte counts and normal CRP levels but renal parameters were suggestive of intrinsic renal failure. Peritoneal dialysis and non-invasive ventilation were started. The condition of the child improved and he was weaned from ventilator after 4 days. The renal parameters normalized after 20 cycles of dialysis.Blood and urine cultures were negative.Post-dialysis the child developed polyuria with a daily urine output $>8 \mathrm{~mL} / \mathrm{kg} /$ day. The infant continued to have polyuria inspite of measures to decrease urine output. The infant developed metabolic alkalosis despite acute kidney injury and polyuria. The blood pressures were in normal range. The urine examination showed, red blood cells, granular casts and proteinuria. Urinary electrolytes values showed urine osmolality $-133.2 \mathrm{mOsm} / \mathrm{kg}$ (normal $500-850$ $\mathrm{mOsm} / \mathrm{kg}$ ), urinary chloride $-66 \mathrm{mEq} / \mathrm{L}$ (normal $<10 \mathrm{mEq} / \mathrm{L}$ ), and spot calcium creatinine ratio - 2.96:1.0 (normal $<0.86: 1$ ). Serum calcium,vitamin D and parathyroid hormone levels were within normal range. Ultrasonography of kidney and bladder showed calcifications in apex of medullary pyramids suggesting bilateral medullary nephrocalcinosis. We diagnosed our case as type 2 Bartter syndrome.

The classical Bartter syndrome (type 3) is perinatal in onset and presents with polyhydramnios, neonatal salt wasting and recurrent episodes of dehydration. Antenatal Bartter syndrome (type 1,2 and 4) typically manifests in infancy with severe phenotype compared to the classical syndrome [2]. The biochemical features reflect defect in sodium, chloride and potassium transporter on ascending limb of loop of Henle [3].

Various genes are associated with Bartter syndrome [4]; MAGED2 mutation described recently is associated with transient Bartter syndrome which starts antenatally with severe phenotype and usually resolves by six weeks of age. Our case presented at around six weeks with acute kidney injury without hypomagnesemia [5]. The diagnosis of Bartter syndrome in neonate or infant is suggested by severe hypokalemia, hypochloremia and metabolic alkalosis. Hypercalciuria is typical and nephrocalcinosis is seen resulting from hyper- 